\title{
Sensitivity Analysis of a Portable Microwave Breast Cancer Detection System
}

\author{
Muhammad Masud Rana1, Debarati Nath', Stephen Pistorius ${ }^{1,2,3}$ \\ ${ }^{1}$ Biomedical Engineering Program, University of Manitoba \\ ${ }^{2}$ Department of Physics and Astronomy, \\ ${ }^{3}$ Department of Radiology, and Research Institute of Oncology \& Hematology, University of Manitoba \\ Winnipeg, MB, R3T2N2, Canada \\ ranamm@myumanitoba.ca; nathd1@myumanitoba.ca; stephen.pistorius@umanitoba.ca
}

\begin{abstract}
A prototype portable breast microwave sensing (BMS) system for early breast cancer detection has been developed in our lab. In this paper, we provide preliminary results for the response of the antenna sensor array to a point scatterer, using both simulation and experiment. The portable system uses a horn antenna to transmit frequencies from $1.5 \mathrm{GHz}$ to $6 \mathrm{GHz}$ and a sensor array using thirteen patch antennas. The optimal separation of each antenna in the receiver array was calculated to be $4 \mathrm{~mm}$ based on an envelope correlation coefficient of 0.37 . The BMS system, including the horn antenna, was designed in CST Microwave Studio to mimic the experimental setup. This study compares the E-field characteristics and DC voltages for each sensor, using simulation and experimental results, for both a free air system and with an Aluminum rod placed at different positions in the scanning plane. A $13 \times 10$ array of geometric correction constants was calculated from the simulated E-field. The range of difference between the simulated and experimental results was $-4 \%$ to $3 \%$ for open space conditions and $\pm 20 \%$ when an Aluminum rod was placed at different positions in the scanning plane. The preliminary results are promising and provide some insight as to where improvements must be made to enhance the detection ability of the portable system.
\end{abstract}

Keywords: Portable breast microwave sensing (BMS) system, Breast Cancer, sensor array, horn antenna, E-field, DC voltage, geometric constant.

\section{Introduction}

Breast cancer accounts for $25 \%$ of all new cancer cases for women in Canada, and while the 5-year survival rate for breast cancer in Canada is over 87\%, worldwide, more women die from breast cancer than any other cancer [1]. Detection of early-stage cancer can increase overall survival [2], but X-ray mammography, the current standard for early breast cancer detection, requires breast compression, uses ionizing radiation, and is not ideally suited to the rigors of screening in remote locations and developing countries [3]. Microwave imaging is a promising non-ionizing and cost-effective technique for detecting breast cancer [4]. Its benefits include a contrast between healthy and malignant breast tissue of nearly 2:1 using model-based image reconstruction techniques, relative safety, and does not require breast compression [4].

Several microwave imaging systems have been developed, including Dartmouth's active microwave tomography system operating in the frequency domain from $300 \mathrm{MHz}$ to $3 \mathrm{GHz}$. They use two-dimensional (2D) images obtained by a set of 16 monopole antennas for detecting breast cancer [5]. Other systems have utilized rotating horn or Vivaldi antennas in air or liquid coupling mediums [6]. To localize tumors in the breast, various algorithms, including confocal microwave imaging techniques, multiplicative delay and sum, and holographic approaches, have been used [6]. A spintronic sensorbased radar imaging system was proposed to detect breast cancer by measuring the amplitude and phase from the backscattering signal [7]. During a scan, the transmitter and receiver were mechanically re-positioned over a 2D rectangular aperture to reconstruct the image of the target object [7].

However, most systems are designed for use in a clinical environment and require expert human intervention to interpret the image and decide if a tumor is present. For use in remote regions or developing countries, another approach is needed. Sacristan and Pistorius proposed a simulation-based portable system that would use machine learning to detect the presence of a breast lesion. They simulated a portable prototype system that consisted of a rotating chamber with a point like microwave source and 12 microwave sensors and evaluated different classifiers to determine which approach more 
accurately determined the presence of a lesion in simulated phantom datasets [8]. However, the prototype device still needs to be experimentally validated, and the sensitivity and specificity of detection could be improved.

As part of an ongoing project to improve the existing portable system, we have used both simulation and experiment to carry out a preliminary study investigating the response of the antenna sensor array to a point scatterer as a function of the frequency of the microwave signal and the receiver antenna position. Using this approach, we compared the E-field obtained from the simulation with the measured output voltages from the patch antenna sensors.

\section{Methods}

\subsection{Portable Microwave Sensing System}

The proposed microwave sensing system is shown in Fig. 1. The sensing system, which operates from $1.5 \mathrm{GHz}$ to $6 \mathrm{GHz}$, includes a transmitter, receiver, radio frequency (RF) detector, and switching circuit. A commercial horn antenna ( 2 to $20 \mathrm{GHz}$ ) and patch antennas were used as the microwave transmitter and receiver, respectively. The receiver antennas were separated from the transmitting antenna by a distance of $(20.00 \pm 0.05) \mathrm{cm}$. The receiver antenna array consisted of thirteen (13) microstrip patch antennas (1.5 to $6 \mathrm{GHz})$ installed on a semi-circular platform. The dimension of each patch antenna was $47.0 \times 17.6 \mathrm{~mm}$. Seven RF detectors performed the rectification of the received signal. Each RF detector has two (2) channels with a bandwidth of $1 \mathrm{MHz}$ to $10 \mathrm{GHz}$, and the switching circuit consists of a 16-channel analog multiplexer feeding an Arduino microprocessor. The rectification voltage was measured using a Keysight Digital Multi-meter and recorded using BenchVue Software.

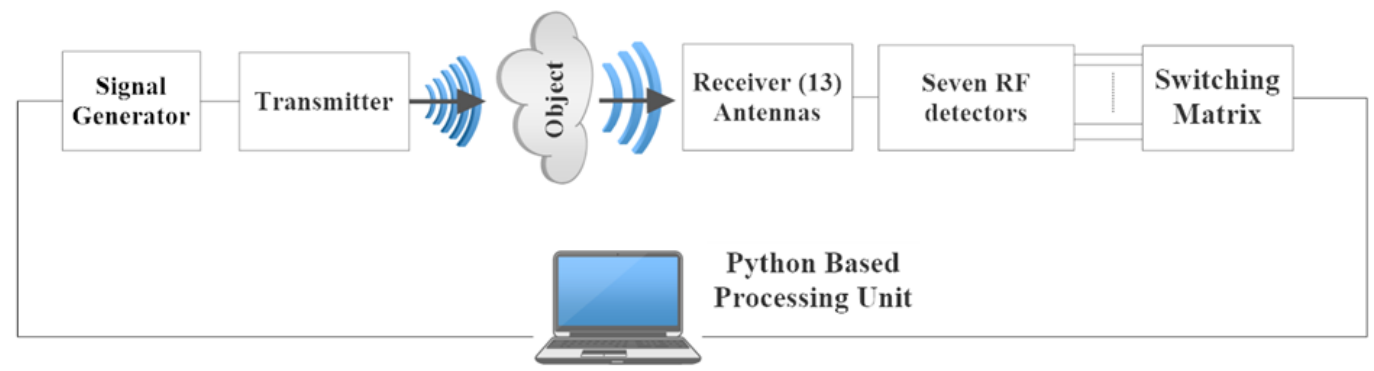

Fig. 1: Schematic view of the portable microwave detection system

\subsection{Proposed Receiver Antenna Array}

Mutual coupling is a common factor in an antenna array design. The optimum nonzero value of mutual coupling will lead to maximum array gain for an antenna array [9]. The Envelope Correlation Coefficient (ECC) is one metric used to determine the effect of mutual coupling in the antenna array [9]. The acceptable limit of ECC is less than 0.5 for a UWB-MIMO antenna system [10]. The ECC can be obtained from the scattering $(S)$ parameters for an array of two antennas [10]. Using Scattering parameters, the ECC can be expressed, as shown in Eq (1) and equation 2 is the simplified form of equation 1.

$$
\begin{aligned}
& \mathrm{ECC}=\frac{\left|S_{11}^{*} S_{12}+S_{21}^{*} S_{22}\right|^{2}}{\left(1-\left|S_{11}\right|^{2}-\left|S_{21}\right|^{2}\right)\left(1-\left|S_{22}\right|^{2}-\left|S_{12}\right|^{2}\right)} \\
& \mathrm{ECC}=4 \times \frac{\left|S_{11}\right|^{2}\left|S_{22}\right|^{2}}{\left(1-\left|S_{11}\right|^{2}-\left|S_{21}\right|^{2}\right)\left(1-\left|S_{22}\right|^{2}-\left|S_{12}\right|^{2}\right)}
\end{aligned}
$$

To maximize the number of receiver antennas in the array, the optimal spacing between the patch antennas was found by minimizing both the ECC and the antenna spacing.

\subsection{Simulation of Antenna Design}

CST Microwave Studio was used to simulate the portable microwave system. To establish a comparison between the measured data from the portable microwave system and the simulation results, a double ridged horn antenna (DRHA) was designed to match the actual horn antenna. The proposed antenna has four (4) sections: the horn, the 
waveguide, the ridges, and the feed. The waveguide section was rectangular, whereas the horn section followed a standard horn shape. The designed horn antenna is shown in Fig. 2, having an axial length (Lf) of $90 \mathrm{~mm}$, an aperture width (Wa) of $104 \mathrm{~mm}$, an aperture height (Ha) of $70 \mathrm{~mm}$, a waveguide height $(\mathrm{Hg})$ of $40 \mathrm{~mm}$, and a waveguide width (Wg) of $41 \mathrm{~mm}$.

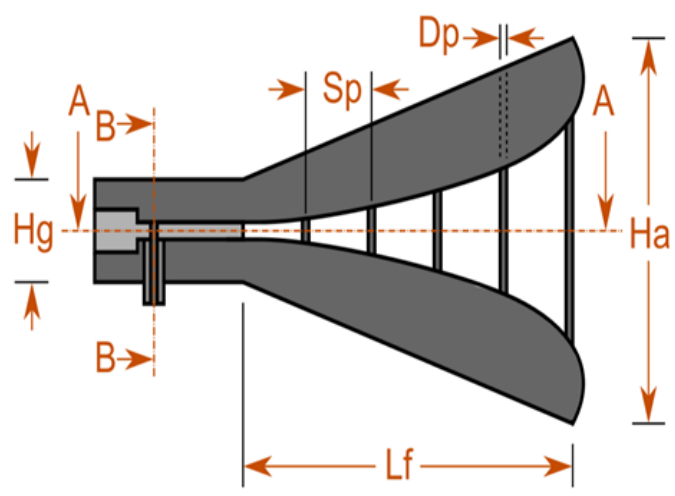

(a)

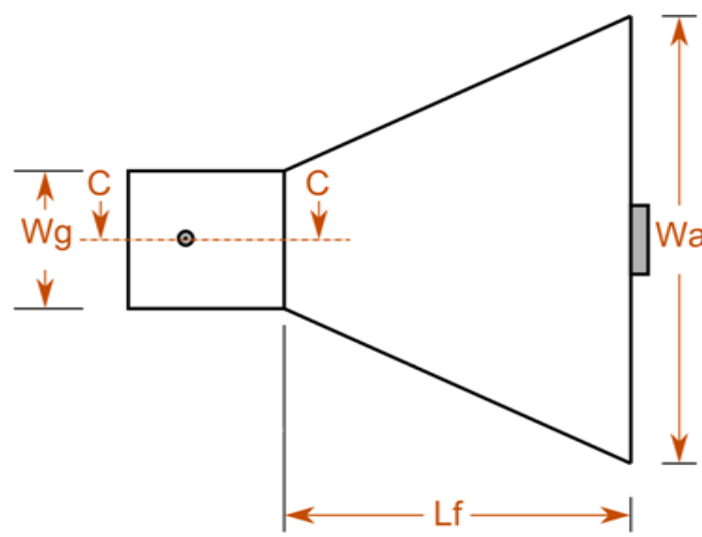

(b)

Fig. 2: (a) Side view of a horn antenna and (b) top view of the horn antenna.

The typical ridge curve of the conventional DRHA uses a straight line and an exponential type curve [11]. The designed antenna used a Bezier curve instead of an exponential curve, as the Bezier curve could be more finely controlled to obtain the appropriate impedance matching. The coaxial feed guides the energy through the lower ridge into the gap between the ridges, in a way that the outer conductor was connected to the lower ridge, and the inner conductor connected to the upper ridge. The proposed antenna matched $50 \Omega$ at the feed to $377 \Omega$ at the aperture of the antenna using the following impedance matching equations [12]- [13].

$$
Z(k)=z_{0} e^{k z}
$$

where $z$ is the distance from the waveguide aperture, $\mathrm{L}$ is the axial length of the flare section, and $\mathrm{k}$ is determined by the following equation.

$$
k=\frac{1}{L} \ln \frac{z_{L}}{z_{o}}
$$

Here, $\mathrm{z}_{0}$ and $\mathrm{z}_{\mathrm{L}}$ are the characteristic impedances of the double-ridged rectangular waveguide and the free space, respectively. The back cavity of the designed antenna is rectangular. While the dimensions of the back cavity play a vital role in return loss and the pattern behavior of the antenna, no analytical process was found to design this section [14]. The dimensions of the back cavity for the proposed antenna were empirically determined by changing the parameters of the back cavity until the desired return loss was obtained. For the desired return loss, the dimension of the back cavity had a width of $22.77 \mathrm{~mm}$, a length of $7.00 \mathrm{~mm}$, and a height of $11.50 \mathrm{~mm}$. The transition between the coaxial probe and the double-ridged rectangular waveguide was also taken into consideration during the design, in order to achieve a low Voltage standard wave ratio (VSWR) [13].

\subsection{Setup for the Measurement of a Point like Scatter}

For the portable microwave system, the $\mathrm{x}$-axis was taken to be $11 \mathrm{~cm}$ from the central axis of the transmitting antenna and $9 \mathrm{~cm}$ from the midpoint of the receiver array, as shown in Fig. 3. The positions of the antennas in the receiver array system are symmetrical, with antenna 7 lying on the $\mathrm{x}=0$ axis, and antennas 1 and 13 in the array being symmetrically positioned at $\mathrm{y} \approx 0$. An aluminum $(\mathrm{Al})$ rod of $6.45 \mathrm{~mm}$ diameter was used to investigate the response of the system to a 
point-like scattered as a function of frequency and position. In this work, we compared the measured and simulation results for 3 points on the $\mathrm{x}$-axis as a function of antenna position, for frequencies from 1.5 to $6 \mathrm{GHz}$ with intervals of GHz. The target of interest was placed at $(-6 \mathrm{~cm}, 0 \mathrm{~cm}),(0 \mathrm{~cm}, 0 \mathrm{~cm})$, and $(6 \mathrm{~cm}, 0 \mathrm{~cm})$ in the scanning plane. We discussed the results for 2.5 and $4 \mathrm{GHz}$ frequency points in the results and discussion section.

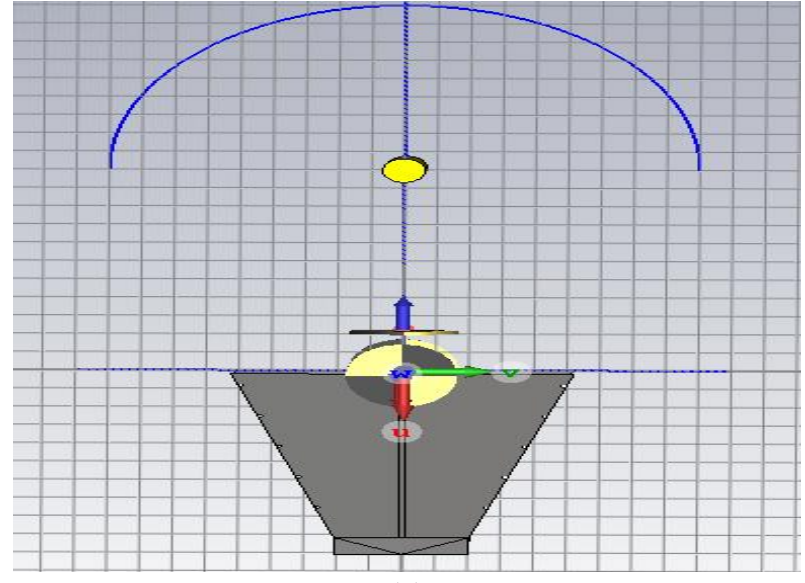

(a)

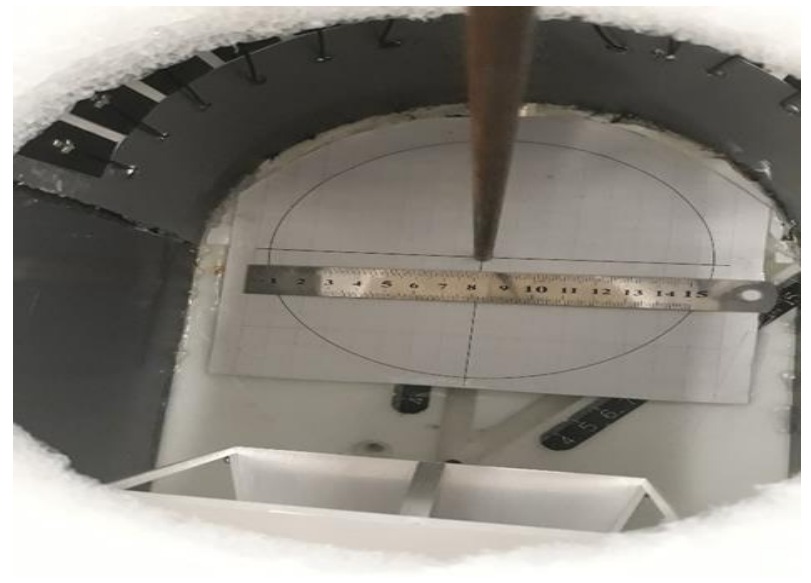

(b)

Fig. 3: (a) Simulation with Aluminium (Al) Rod and (b) Experimental setup for the portable system.

\section{Result and Discussion}

\subsection{Calculation of Optimal Separation of Antennas in Receiver Array}

The normalized maximum ECC for separation distances of 0 to $15 \mathrm{~mm}$ is shown in Fig. 4. Since the ideal sensor array would have zero spacing and an $\mathrm{ECC}=0$, the optimal spacing was obtained by selecting a spacing that minimized both ECC and spacing using the shortest distance to $(E C C=0, N D D=0)$ as illustrated in Fig. 4. At this point the calculated ECC was 0.37 , which is less than the recommended constraint of 0.50 , and the optimal separation between each antenna in the receiver array was $4 \mathrm{~mm}$.

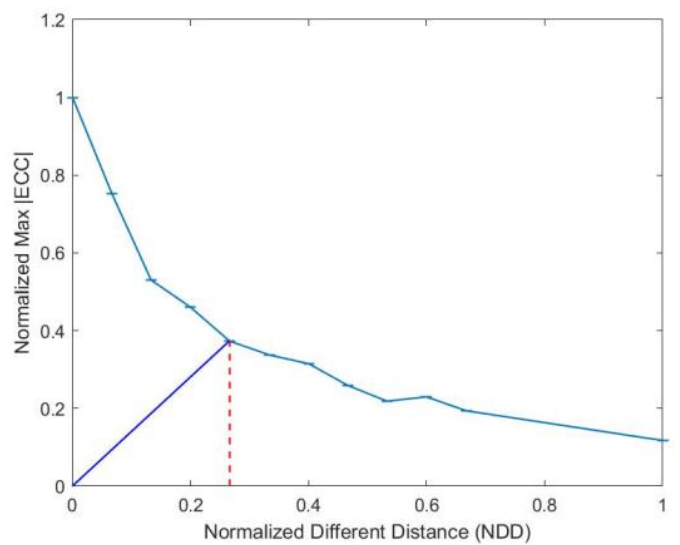

Fig. 4: Output from the parametric study of the Envelope Correlation Coefficient (ECC), normalized to the maximum ECC value of 0.99 , for distances from 0 to $15 \mathrm{~mm}$ between two antennas, normalized to the maximum $15 \mathrm{~mm}$ spacing (NDD).

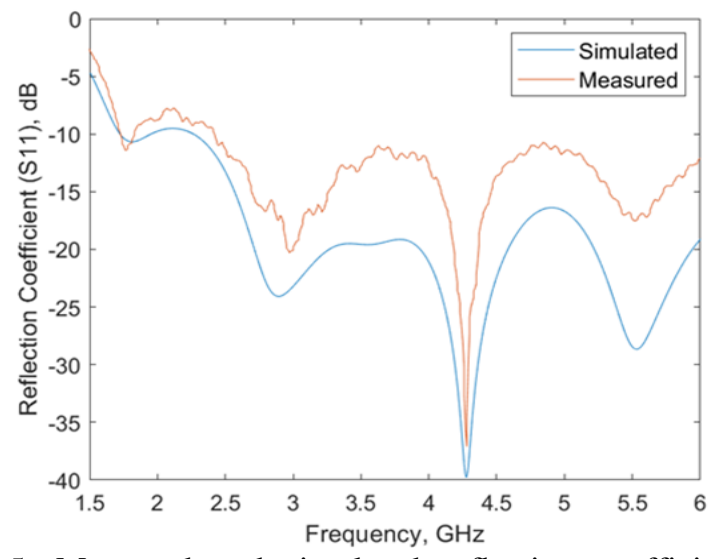

Fig. 5: Measured and simulated reflection coefficient. The resonant frequency occurred at $4.30 \mathrm{GHz}$ frequency for both simulated and measured reflection coefficient. 


\subsection{Comparison between the Simulated Antenna and the Antenna Used in Experiment}

The results of the simulated and measured reflection coefficients for the horn antenna were plotted in Fig. 5. The measured and simulated results had a bandwidth agreement of $90 \%$ at $-10 \mathrm{~dB}$, and the resonant frequency occurred at 4.3 $\mathrm{GHz}$ frequency for both simulated in CST and measured in the laboratory. The VSWR of the simulated antenna was less than 2 within the bandwidth. The radiation patterns were also compared between the simulation and the measurement and agreed to within $96 \%$.

\subsection{Measurement of a Point like Scatter}

The open space measured output voltage had a linear relationship to the input power given by Eq. (5), with some typical parameters and correlation coefficients shown in Table 1 for antennas 1 and 13 at $2.5 \mathrm{GHz}$ and $4.0 \mathrm{GHz}$. While the gain of each symmetrically positioned antenna was approximately equal, the bias factors at $0 \mathrm{dBm}$ input power, shown in Fig. 6 (a), were different.

$$
V(\text { voltage })=(\mathrm{B} \pm \Delta \mathrm{B})+(\mathrm{A} \pm \Delta A) * P(\text { Power }, d B m)
$$

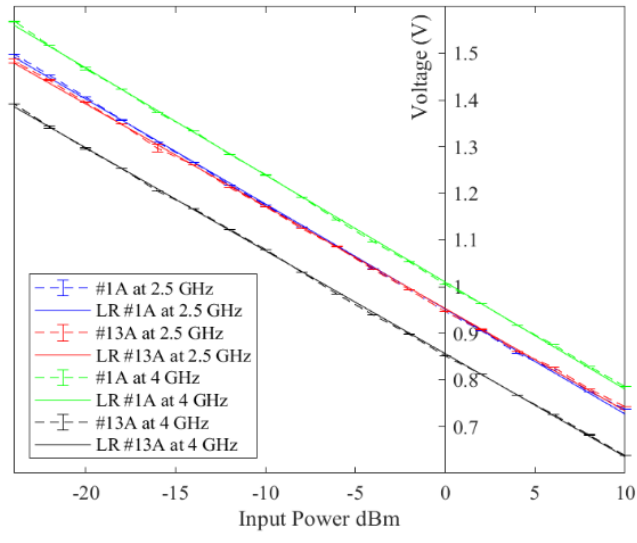

(a)

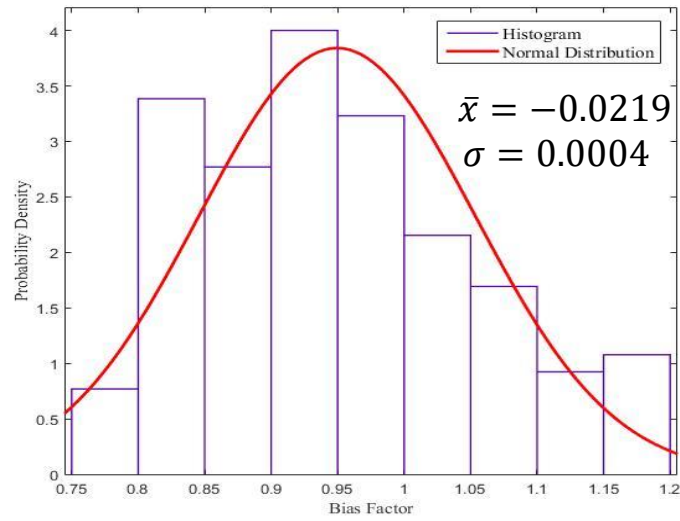

(b)

Fig. 6: (a) Dashed lines represent measured voltage, and solid lines represent the corresponding regression line for antenna number 1 and 13 with respect to different input power $(\mathrm{dBm})$ at $2.5 \mathrm{GHz}$ and $4 \mathrm{GHz}$. Each symmetrical sensor has approximately the same gain but different weight or bias factor at $0 \mathrm{dBm}$ input power. (b) Bias factor distribution for 13 number antennas with 10 frequency points from $1.5 \mathrm{GHz}$ to $6 \mathrm{GHz}$ with an interval of $0.5 \mathrm{GHz}$.

According to the linear relationship, all sensors had a negative gain ranging from $-0.0230 \mathrm{dBm} / \mathrm{V}$ to $-0.0210 \mathrm{dBm} / \mathrm{V}$ with a mean and two standard deviations of $-0.0219 \pm 0.0007 \mathrm{dBm} / \mathrm{V}$. Fig. 6 (b) shows the probability distribution of the bias factor for all antennas at 10 frequency points. The bias factor for $95 \%$ of the expected population $(\bar{x} \pm 2 \sigma)$, ranged from $0.752 \mathrm{~V}$ to $1.167 \mathrm{~V}$.

Table 1: Linear parameters with two standard deviation and correlation coefficients for antennas 1 and 13 at $2.5 \mathrm{GHz}$ and 4 $\mathrm{GHz}$.

\begin{tabular}{|c|c|c|c|c|c|}
\hline $\begin{array}{c}\text { Frequency } \\
(\mathrm{GHz})\end{array}$ & $\begin{array}{l}\text { Antenna } \\
\text { Number }\end{array}$ & $\begin{array}{c}\text { Gain, A } \\
\text { (dBm/Volts) }\end{array}$ & $\begin{array}{c}\text { Bias Factor, B } \\
\text { (Volts) }\end{array}$ & $\begin{array}{l}\text { correlation } \\
\text { coefficient }\end{array}$ & RMSE \\
\hline \multirow{2}{*}{2.5} & 1 & $-0.0231 \pm 0.0002$ & $0.952 \pm 0.004$ & \multirow{2}{*}{0.9998} & \multirow{2}{*}{0.0044} \\
\hline & 13 & $-0.0222 \pm 0.0004$ & $0.946 \pm 0.007$ & & \\
\hline \multirow{2}{*}{4} & 1 & $-0.0231 \pm 0.0001$ & $1.011 \pm 0.003$ & \multirow{2}{*}{0.9998} & \multirow{2}{*}{0.0046} \\
\hline & 13 & $-0.0221 \pm 0.0001$ & $0.856 \pm 0.002$ & & \\
\hline
\end{tabular}


From the approximation of far-field antenna behavior, the electric field strength $E$ depends on the square root of input power in watts in the following $\mathrm{Eq}(6)$. Where the electric field strength $E(\mathrm{~V} / \mathrm{m})$, Horn antenna gain $G(\mathrm{~dB})$, Characteristics Impedance $Z_{0}(\Omega)$, Input power $P(\mathrm{~W})$, and distance between receiver antennas $d(\mathrm{~m})$. Geometric $\mathrm{C}$ is an array, which varies on 13 number of receiver antennas $(A n t)$, and 10 frequency points $(F)$ from $1.5 \mathrm{to} 6 \mathrm{GHz}$ intervals of $0.5 \mathrm{GHz}$. From the geometric constant in Eq (7), we can calculate the received power.

$$
\begin{aligned}
& E(A n t, F)=\sqrt{\frac{Z_{0} G(F)}{4 \pi d^{2}}} \sqrt{P} \\
& E(A n t, F)=C(A n t, F) \sqrt{P}
\end{aligned}
$$

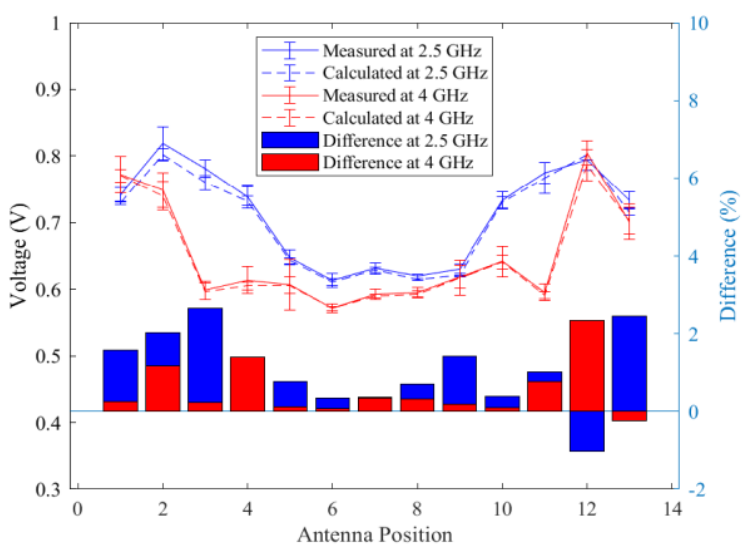

(a)

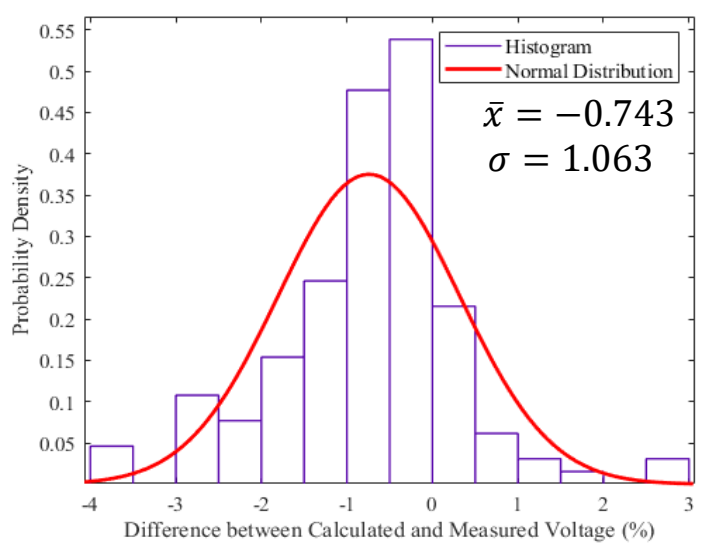

(b)

Fig. 7: (a) Solid (blue and red) lines represent measured voltage and dashed (blue and red) lines represent calculated voltage from simulated E-filed patterns for open space conditions at $2.5 \mathrm{GHz}$ and $4 \mathrm{GHz}$ frequency. The blue and red bars represent the percentage difference between the calculated and measured voltages. (b) The distribution of the percentage difference between the simulation and experimental measurements for 13 antennas with 10 frequency points from $1.5 \mathrm{GHz}$ to $6 \mathrm{GHz}$ with an interval of $0.5 \mathrm{GHz}$.

The measured and calculated voltages from the simulated E-field pattern for the open space conditions at different frequencies are plotted in Fig. 7 (a). The solid blue line represents the measured voltage for the receiver antennas, and the dashed line represents the voltage derived from the simulated E-field at the receiver antennas. Fig. 7 illustrates that the measured and calculated voltages agree to within $96 \%$ for all receiver antennas. For example, at a frequency of 2.5 $\mathrm{GHz}$, the maximum error occurred at antenna 3, while at a frequency of $4 \mathrm{GHz}$, the maximum error occurred at antenna 12 . The differences between the measured and calculated voltages at the receiver antennas, varied from $-2.9 \%$ to $1.4 \%$, within a $95 \%$ confidence interval.

The E-fields at the receiver antenna array shown in Fig. 8 followed the additive and subtractive properties of the transmitted and scattered signals for different $\mathrm{Al}$ rod positions. Fig. 8 shows the normalized electrical field at receiver antenna positions for the $\mathrm{Al}$ rod positions in the scanning plane with respect to the open space electrical field at the receiver antenna positions. The normalized electrical field at antenna 1 when the $\mathrm{Al}$ rod was at the $(-6 \mathrm{~cm}, 0 \mathrm{~cm})$ position, was the same as the normalized electric field at antenna 13 for the $\mathrm{Al} \operatorname{rod}(6 \mathrm{~cm}, 0 \mathrm{~cm})$ position at $2.5 \mathrm{GHz}$ and $4 \mathrm{GHz}$ frequency. Fig. 8 also illustrates that the normalized electric fields at antenna 1 and antenna 13 are the same for the $\mathrm{Al}$ rod at the $(0 \mathrm{~cm}, 0 \mathrm{~cm})$ position. 


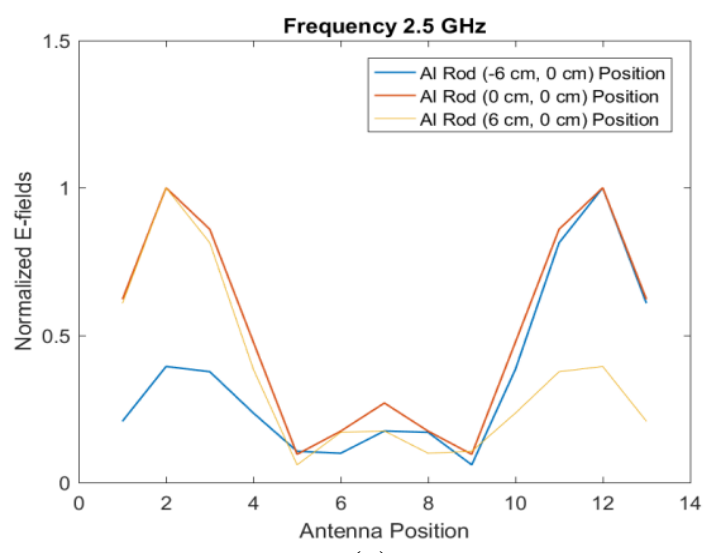

(a)

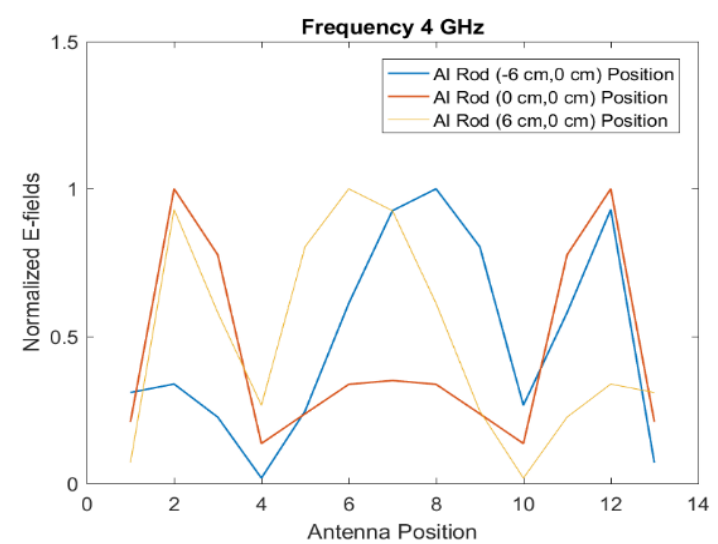

(b)

Fig. 8: Normalized simulated E-fields' for different positions of Aluminum (Al) rod at $2.5 \mathrm{GHz}$ and $4 \mathrm{GHz}$.

Fig. 9 (a) illustrates the measured and calculated voltages from the simulated E-field for the Aluminum (Al) rod at point $(-6 \mathrm{~cm}, 0 \mathrm{~cm})$ at frequencies of $2.5 \mathrm{GHz}$ and $4 \mathrm{GHz}$. The measured and calculated voltages for all receiver antennas were not equal because of slight differences in the experimental setup and simulation environment. Fig. 9 (b) shows the percentage distribution of the differences between the calculated and measured voltages for the three rod positions $(-6 \mathrm{~cm}$, $0 \mathrm{~cm}),(0 \mathrm{~cm}, 0 \mathrm{~cm})$ and $(6 \mathrm{~cm}, 0 \mathrm{~cm})$. The error distributions were normally distributed. A one-way ANOVA for nonequal variance using Welch's test showed strong evidence for the null hypothesis $(\mathrm{p}=0.951>0.05)$, which concludes that the mean differences for the three different rod positions were not significant. The differences in the measured and calculated voltages for receiver antennas from $1.5 \mathrm{GHz}$ to $6 \mathrm{GHz}$ were approximately $\pm 20 \%$ at the $95 \%$ confidence interval.

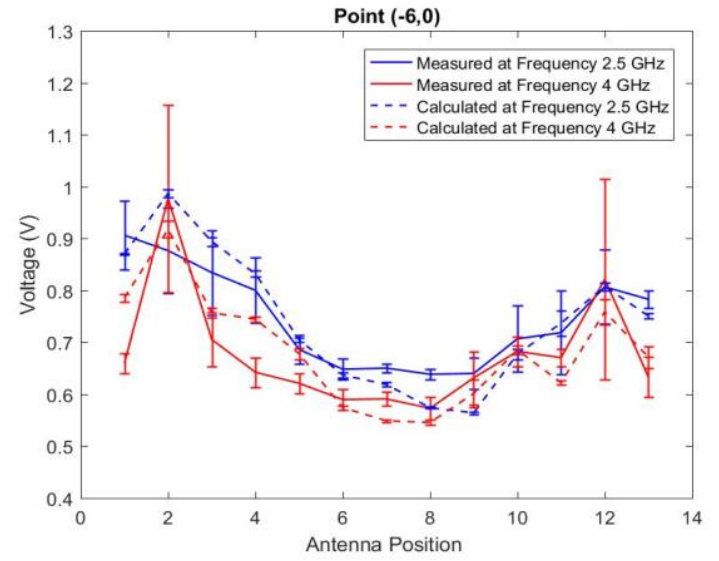

(a)

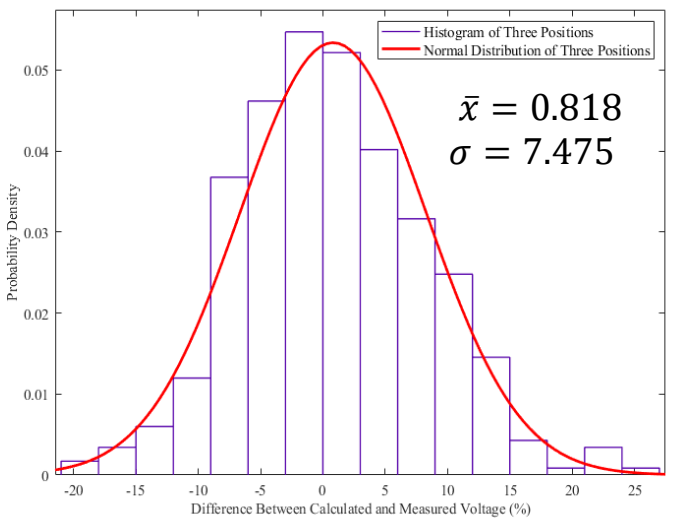

(b)

Fig. 9: (a) Solid (blue and red) lines represent the measured voltage and dashed (blue and red) lines represent the calculated voltage from simulated E-field patterns for the Aluminum (Al) rod at point $(-6 \mathrm{~cm}, 0 \mathrm{~cm})$, and at $2.5 \mathrm{GHz}$ and $4 \mathrm{GHz}$ frequencies. (b) A histogram of the percentage of differences between simulation and experimental measurements for $\mathrm{Al}$ rod at $(-6 \mathrm{~cm}, 0 \mathrm{~cm}),(0 \mathrm{~cm}, 0$ $\mathrm{cm}),(6 \mathrm{~cm}, 0 \mathrm{~cm})$ position and combination of three positions for 13 number antennas with 10 frequency points from $1.5 \mathrm{GHz}$ to 6 $\mathrm{GHz}$ with an interval of $0.5 \mathrm{GHz}$, fitted with a normal distribution.

\section{Conclusion}

This work presents some preliminary findings on the response of a portable breast cancer detection system to a pointlike scatterer as a function of the frequency of the transmitted microwave signal and the position of the sensors in the receiver array. A semi-circular antenna array using thirteen microstrip patch antennas with a $4 \mathrm{~mm}$ gap between the sensors 
was used. The portable system was simulated in CST Microwave Studio. The simulated E-fields were, as expected, found to be symmetrical both for the open field and for changes in the position of the Al rod. The measured DC voltages from the sensor as a function of the antenna positions were not well correlated with the simulated E-field, because of the different channel bias factor of the RF detectors. For open space comparisons, the calculated and measured voltage were approximately the same, with a $96 \%$ agreement between the simulated and experimental results. For a point like scatterer, the calculated and measured voltage had differences of between $\pm 20 \%$. Improving the agreement between the simulated and experimental measurements will require a more accurate simulation of the experimental conditions. Following this, a combination of simulation and measurements will be used to develop a machine learning approach to breast cancer detection for this system.

\section{Acknowledgements:}

Funding from the University of Manitoba, National Science and Engineering Research Council and the CancerCare Manitoba Foundation.

\section{References}

[1] Canadian Cancer Statistics Advisory Committee. Canadian Cancer Statistics, 2019. Toronto, ON: Canadian Cancer Society; 2019. Available at: cancer.ca/Canadian-Cancer-Statistics-2019-EN. September 2019 ISSN 0835-2976 [Accessed: 19-Jan-2020].

[2] D. van der Waal, A. L. M. Verbeek, G. J. den Heeten, T. M. Ripping, V. C. G. Tjan-Heijnen, and M. J. M. Broeders, "Breast cancer diagnosis and death in the Netherlands: a changing burden," Eur. J. Public Health, vol. 25, no. 2, pp. 320-324, Apr. 2015.

[3] E. K. J. Pauwels, N. Foray, and M. H. Bourguignon, "Breast Cancer Induced by X-Ray Mammography Screening? A Review Based on Recent Understanding of Low-Dose Radiobiology," Med. Princ. Pract., vol. 25, no. 2, pp. 101109, Feb. 2016.

[4] J. Bourqui, J. M. Sill, and E. C. Fear, "A Prototype System for Measuring Microwave Frequency Reflections from the Breast," International Journal of Biomedical Imaging, vol. 2012, pp. 1-12, 2012.

[5] E. C. Fear, S. C. Hagness, P. M. Meaney, M. Okoniewski, and M. A. Stuchly, "Enhancing breast tumor detection with near-field imaging," IEEE Microw. Mag., vol. 3, no. 1, pp. 48-56, Mar. 2002.

[6] E. C. Fear, X. Li, S. C. Hagness, and M. A. Stuchly, "Confocal microwave imaging for breast cancer detection: localization of tumors in three dimensions," IEEE Trans. Biomed. Eng., vol. 49, no. 8, pp. 812-822, Aug. 2002.

[7] L. Fu W. Lu, D. Rodriguez-Herrera, D. Flores-Tapia, Y.S. Gui, S. Pistorius, C.-M. Hu, "Microwave radar imaging using a solid state spintronic microwave sensor," Appl. Phys. Lett., vol. 105, no. 12, p. 122406, Sep. 2014.

[8] J. Sacristán and S. Pistorius, "A Comparison of Classifiers for Detecting Tumours Using Microwave Scattering in Numerical Breast Models," CMBES Proc., vol. 40, 2017.

[9] C.-X. Mao and Q.-X. Chu, "Compact Coradiator UWB-MIMO Antenna with Dual Polarization," IEEE Trans. Antennas Propag., vol. 62, no. 9, pp. 4474-4480, Sep. 2014.

[10] G. Srivastava and A. Mohan, "Compact MIMO Slot Antenna for UWB Applications," IEEE Antennas Wirel. Propag. Lett., vol. 15, pp. 1057-1060, 2016.

[11] Z. Yue, Y. Liu, and S. Gong, "A novel 2-18 GHz double-ridged horn antenna with simple structure," Microw. Opt. Technol. Lett., vol. 60, no. 7, pp. 1787-1794, 2018.

[12] M. Solis Nepote, D. R. Herrera, D. F. Tapia, S. Latif, and S. Pistorius, "A comparison study between horn and vivaldi antennas for 1.5-6 GHz breast microwave radar imaging," in The 8th European Conference on Antennas and Propagation (EuCAP 2014), 2014, pp. 59-62.

[13] A. R. Mallahzadeh and A. A. Dastranj, "Double-Ridged Conical Horn Antenna for 2-18 GHz," Electromagnetics, vol. 28, no. 6, pp. 450-461, Aug. 2008.

[14] M. Ghorbani and A. Khaleghi, "Double ridged horn antenna designs for wideband applications," in 2011 19th Iranian Conference on Electrical Engineering, 2011, pp. 1-4. 